\title{
TWO THEOREMS IN THE COMMUTATOR CALCULUS ${ }^{1}$ )
}

\author{
BY \\ HER MANN V. WALDINGER
}

\begin{abstract}
Let $F=\langle a, b\rangle$. Let $F_{n}$ be the $n$th subgroup of the lower central series. Let $p$ be a prime. Let $c_{3}<c_{4}<\cdots<c_{z}$ be the basic commutators of dimension $>1$ but $<p+2$. Let $P_{1}=(a, b), P_{m}=\left(P_{m-1}, b\right)$ for $m>1$. Then $\left(a, b^{p}\right) \equiv \prod_{i=3}^{z} c_{i}^{n_{1}} \bmod F_{p+2}$. It is shown in Theorem 1 that the exponents $\eta_{i}$ are divisible by $p$, except for the exponent of $P_{p}$ which $=1$.

Let the group $\mathscr{G}$ be a free product of finitely many groups each of which is a direct product of finitely many groups of order $p$, a prime. Let $\mathscr{G}^{\prime}$ be its commutator subgroup. It is proven in Theorem 2 that the " $\mathscr{G}$-simple basic commutators" of dimension $>1$ defined below are free generators of $\mathscr{G}^{\prime}$.
\end{abstract}

I. Introduction. This paper is the result of research on the factor groups of the lower central series of groups, $\mathscr{G}$, defined below before the statement of Theorem 2. It was shown in [8] that these factor groups have bases which are images of special words in "fundamental commutators." The author has now succeeded in showing that there are such bases which consist of images of specific powers of "fundamental commutators." The present proof of this result is very complicated, but it requires Theorems 1 and 2 below which are of interest in themselves.

In a classical paper [4], P. Hall first established the following identity (1.1): Let $F=F_{1}$ be the free group with $a, b$ as free generators. Let $F_{n}$ be the $n$th subgroup of the lower central series of $F$. Let $p$ be a prime. Let $c_{1}=b<c_{2}=a<\cdots<c_{q}$ be the basic commutators of dimension $\leqq p-1$. (For definitions of the basic commutators see [3].) Then

$$
(a b)^{p}=\left(\prod_{i=1}^{q} c_{i}^{\varepsilon_{i} p}\right) Q
$$

Received by the editors May 19, 1971.

AMS 1970 subject classifications. Primary 20E30, 20F35, 20F40; Secondary 20E35, 20F05, $20 \mathrm{~K} 15$.

Key words and phrases. Group, subgroup, free product of groups, direct product of groups cyclic group, abelian group, free group, free abelian group, finitely generated group, associative algebra, generator, free generator, relator, order of group, prime $p$, commutator, basic commutator, commutator subgroup, 2nd commutator subgroup, lower central series, subgroup of the lower central series, dimension, indeterminate, commuting indeterminates, formal infinite sum, homogeneous polynomial, degree of a polynomial, linear independence, integral coefficient, coefficient or exponent divisible by $p$, " $\mathscr{F}$-simple basic commutator", " $\mathscr{G}$-simple basic commutator", abelianized group, faithful representation, collection process, nontrivial relator, Jacobi relator, homomorphism, image under a homomorphism.

( $\left.{ }^{1}\right)$ Work done under NSF grant GP-11348. 
where $\varepsilon_{1}=\varepsilon_{2}=1$, the numbers $\varepsilon_{1}, \varepsilon_{2} \cdots \varepsilon_{q}$ are integers and $Q \in F_{p}$. In this paper we shall give a proof of the related identity (1.2).

THEOREM 1. Let $c_{q+1}<c_{q+2}<\cdots<c_{z}$ be the basic commutators of dimensions $p$ and $p+1$. Let $P_{1}=(a, b)$ and $P_{m}=\left(P_{m-1}, b\right)$ for $m>1$, let $c_{s}=P_{p}$. Then

$$
\left(a, b^{p}\right)=\left(\prod_{i=3}^{z} c_{i}^{\eta_{i}}\right) P
$$

where all the exponents $\eta_{i}$ occurring in (1.2) are divisible by p except for $\eta_{s}$, but $\eta_{s}=1$ and $P \in F_{p+2}$.

Identity (1.2) was proven by Struik [7] using a modification of the proof of (1.1) given in [4]. We shall, however, prove it here by the methods of [6].

In [2], K. W. Gruenberg found a set of free generators for the commutator subgroup, $G^{\prime}$, of the group, $G$, which is the free product of finitely many cyclic groups of finite order. We shall prove here a related result on $\mathscr{G}^{\prime}$, the commutator subgroup of the group

$$
\mathscr{G}=\mathscr{G}(1) * \mathscr{G}(2) * \cdots * \mathscr{G}(s),
$$

where each free factor $\mathscr{G}(i)$ is the direct product of $\left(n_{i}-n_{i-1}\right)$ cyclic groups of order $p$ having generators $c_{n_{i-1}+1}, c_{n_{i-1}+2}, \ldots, c_{n_{i}}$. (Here $O=n_{0}<n_{1}<n_{2}<\cdots<n_{s}=r$.) The role of the " $\mathscr{G}$-simple basic commutators" of dimension $>1$ as generators of $\mathscr{G}^{\prime}$ is discussed in [8]. We shall show here

THEOREM 2. $\mathscr{G}$ ' is freely generated by the "G-simple basic commutators" of dimension >1. (These commutators are defined in the proof below.)

To obtain this theorem we shall first demonstrate that the images of our free generators in the abelianized group $\mathscr{G}^{\prime} / \mathscr{G}^{\prime \prime}$ are free generators. We shall do so by adapting the methods employed by $\mathrm{S}$. Bachmuth [1] in his study of the commutator subgroup of a free metabelian group with finitely many free generators. These methods are based on the faithful matrix representations given by $\mathrm{W}$. Magnus [5]. Finally by making use of standard theorems on abelian groups, free groups and free products of groups, we shall show that our result for $\mathscr{G}^{\prime} \mid \mathscr{G}^{\prime \prime}$ implies Theorem 2.

II. Proof of Theorem 1. Evidently $\left(a, b^{p}\right) \in F_{2}$; hence $\left(a, b^{p}\right)$ can be written uniquely in the form (1.2) by a well-known theorem of P. Hall ([3], [4], [6]). It remains to determine the coefficients $\eta_{i}$. We shall do so by making the standard substitutions [6]

$$
a=1+x, \quad b=1+y,
$$

where $x$ and $y$ are free generators of an associative algebra. We find then easily that

$$
\left(a, b^{p}\right)=\left[(a, b) b^{-1}\right]^{p} b^{p}=(1-Z)^{p}(1+y)^{p}=1+p Y+y^{p}+(-Z)^{p}+O_{2 p}
$$

where $Y$ and $O_{s}$ are formal infinite sums of terms in $x$ and $y, O_{s}$ contains only terms of degree at least $s$ and

$$
Z=y-y^{2}+y x-x y+O_{3} .
$$


To proceed we note that

$$
y^{p}+(-Z)^{p}=\Sigma+\left[1+(-1)^{p}\right] y^{p}+p(-y)^{p+1}+O_{p+2}
$$

where

$$
(-1)^{p+1} \Sigma=\sum_{m=0}^{p-1} y^{m}(x y-y x) y^{p-1-m}
$$

To obtain our result from formulas (2.2)-(2.5) we must now recall two wellknown facts [6]:

(i) If the basic commutator $c_{j}$ has dimension $d$, then

$$
c_{j}=1+\mu\left(c_{j}\right)+O_{d+1}
$$

where $\mu\left(c_{j}\right)$ is a homogeneous polynomial of degree $d$.

(ii) If $c_{u}, c_{u+1}, \ldots, c_{v}$ are the basic commutators of dimension $d$, then $\mu\left(c_{u}\right)$, $\mu\left(c_{u+1}\right), \ldots, \mu\left(c_{v}\right)$ are linearly independent.

From (i) and (ii) we easily find (iii) as a consequence:

(iii) If $i$ is the smallest positive integer so that $\eta_{i}$ is not divisible by $p$ and $c_{i}$ has dimension $\bar{d}$, then the substitution (2.1) yields

$$
\left(a, b^{p}\right)=1+p Y+R+O_{\bar{d}+1}
$$

where $R \neq 0$ and is a homogeneous polynomial of degree $\bar{d}$ so that at least one among its coefficients is not divisible by $p$. We conclude then by (2.2), (2.4) and (2.5) that $\bar{d}=p+1$, also that to establish the truth of the theorem we only need to show that $\mu\left(P_{p}\right)-\Sigma$ is divisible by $p$. Now

$$
\mu\left(P_{p}\right)-\Sigma=\sum_{j=0}^{p-1}\left\{\left[(-1)^{j}\left(\begin{array}{c}
p-1 \\
j
\end{array}\right)+(-1)^{p}\right]\left[y^{j}(x y-y x) y^{p-j-1}\right]\right\}
$$

by a simple computation. Let $I$ and $J$ be integers. We note from the definition of the binomial coefficients that

$$
\begin{aligned}
(-1)^{j}\left(\begin{array}{c}
p-1 \\
j
\end{array}\right) & +(-1)^{p} \\
& =0 \text { for } j=0 \text { and } p>2 \\
& =2 \text { for } j=0 \text { and } p=2, \\
& =((p-1)(p-2) \cdots(p-j)-j !) / j !=p I / j ! \\
\text { for } 0<j \leqq & \quad \text { for } 0<j \leqq p-1, j \text { odd and } p>2, \\
& =-\frac{(p-1)(p-2) \cdots(p-[p-j-1])+(p-j-1) !}{(p-j-1) !}=\frac{p J}{(p-j-1) !} \\
& =0 \text { for } p=2 \text { and } j=1 .
\end{aligned}
$$

It is evident from (2.9) that $\mu\left(P_{p}\right)-\Sigma \equiv 0 \bmod p$. Our theorem has been established. 
III. Proof of Theorem 2. In this proof we shall require the two definitions below which were first given in [8].

DEFINITION 1. The commutator

$$
\left(\ldots\left(\left(c_{j_{1}}, c_{j_{2}}\right), c_{j_{3}}\right), \ldots, c_{j_{\tau}}\right)
$$

is said to be an " $\mathscr{F}$-simple basic commutator" of dimension $\tau>1$ if $c_{j_{1}}, c_{j_{2}}, \ldots, c_{j_{\tau}}$ are all elements of the set of generators $\left\{c_{1}, c_{2}, \ldots, c_{r}\right\}$ so that $j_{1}>j_{2} \leqq j_{3} \leqq \cdots \leqq j_{\tau}$.

Definition 2. The "F्F-simple basic commutator" (3.1) is said to be a " $\mathscr{G}$ simple basic commutator" if it satisfies the following three conditions:

(i) $\left(c_{j_{1}}, c_{j_{2}}\right) \neq 1$ in $\mathscr{G}$.

(ii) If $c_{i}$ occurs $\sigma$ times in the set $\left\{c_{j_{1}}, c_{j_{2}}, \ldots, c_{j_{\tau}}\right\}$, then $1 \leqq \sigma<p$.

(iii) If $\left(c_{j_{1}}, c_{j_{\lambda}}\right)=1$ in $\mathscr{G}$ for $2<\lambda \leqq \tau$, then $j_{\lambda} \leqq j_{1}$.

Having given our definitions we are now ready to proceed with our proof. Let us consider an arbitrary element $w \in \mathscr{G}$; evidently it can be written as

$$
W=c_{j_{1}} c_{j_{2}} \cdots c_{j_{z}}
$$

where the subscripts $j_{1}, j_{2}, \ldots, j_{\tau}$ take the values $1,2, \ldots, r$. For $w \neq 1$, let $\sigma(k, w)$ be the number of times the subscript $k(1 \leqq k \leqq r)$ occurs among $j_{1}, j_{2}, \ldots, j_{\tau}$; for $w=1$ we take every $\sigma(k, w)=0$. It is well known ([3], [6]) that a word, $w \neq 1$, in the free group on $r$ generators $c_{1}, c_{2}, \ldots, c_{r}$ is in the commutator subgroup if and only if every generator occurs in $w$ with "exponentsum" 0 . It is then evident that the element (3.2) is in $\mathscr{G}^{\prime}$ if and only if all $r$ "exponentsums" $\sigma(k, w)$ are divisible by $p$. Applying the collection process ([3], [4]) to such a $w$ and making use of Definition 1 , we conclude easily that $w$ ' is a word in " $\mathscr{F}$-simple basic commutators" of dimension $>1$. But Lemmas 4.2 and 4.4 of [8] assert that every such " $\mathscr{F}$-simple basic commutator" is a word in "Ğ-simple basic commutators" of dimension $>1$.

We have now shown

\section{LeMmA 1. The "G-simple basic commutators" of dimension $>1$ generate $\mathscr{G}^{\prime}$.}

It remains to show that they do so freely. To arrive at this result we shall first demonstrate that their images do so in the abelianized commutator subgroup. For this purpose we shall make use of the matrix representation given by W. Magnus [5].

In particular we shall find a representation of $\overline{\mathscr{G}}=\mathscr{G} / \mathscr{G}^{\prime \prime}$ where $\mathscr{G}^{\prime \prime}=\left[\mathscr{G}^{\prime}, \mathscr{G}^{\prime}\right]$, the second commutator subgroup of $\mathscr{G}$. In this connection we first note that $\mathscr{G}$ is a homomorphic image of $\mathscr{F}$ according to the presentations of the form

$$
\mathscr{F}=\left\langle c_{1}, c_{2}, \ldots, c_{r}\right\rangle, \quad \mathscr{G}=\left\langle c_{1}, c_{2}, \ldots, c_{r} ; S_{1}, S_{2}, \ldots, S_{t}\right\rangle .
$$

Suppose $\mathscr{H}$ is the normal closure in $\mathscr{F}$ of the subgroup generated by all commutators and all relators $S_{1}, S_{2}, \ldots, S_{t}$. Let $\mathscr{H}^{\prime}$ be the commutator subgroup of $\mathscr{H}$; it is obviously normal in $\mathscr{F}$. Let $g_{0}, \tilde{g}$ and $\bar{g}$ be the images in $\mathscr{G}, \mathscr{F} \mid \mathscr{H}^{\prime}$ and $\overline{\mathscr{G}}$ respectively of the element $g \in \mathscr{F}$ under the respective homomorphisms $\mathscr{F} \rightarrow \mathscr{G}$, $\mathscr{F} \rightarrow \mathscr{F} / \mathscr{H}^{\prime}$ and $\mathscr{F} \rightarrow \mathscr{G} \rightarrow \overline{\mathscr{G}}$. Then $\mathscr{F} / \mathscr{H}^{\prime}$ has the faithful matrix representations [5] given by the correspondence 


$$
\tilde{c_{i}} \leftrightarrow X_{i}=\left(\begin{array}{cc}
x_{i} & 0 \\
t_{i} & 1
\end{array}\right)
$$

such that

(i) the $x_{i}, t_{i}$ are $2 r$ commuting indeterminates,

(ii) $x_{i}^{p}=1$ for $i=1,2, \ldots, r$.

Furthermore, the correspondence

$$
\bar{c}_{i} \leftrightarrow X_{i}=\left(\begin{array}{cc}
x_{i} & 0 \\
t_{i} & 1
\end{array}\right)
$$

becomes a faithful matrix representation of $\overline{\mathscr{G}}$ once we impose the additional $t$ conditions that

$$
\bar{S}_{u} \leftrightarrow\left(\begin{array}{ll}
1 & 0 \\
0 & 1
\end{array}\right)
$$

for $u=1,2, \ldots, t$. But the relators $S_{u}$ fall into two categories according to the definition of the group $\mathscr{G}$ given by (1.3):

(I) $S_{u}=c_{i}^{p}$ for $i=1,2, \ldots, r$.

(II) $S_{u}=\left(c_{j}, c_{i}\right)$ for $n_{k-1}<c_{i}<c_{j} \leqq n_{k} \leqq r$ and $k=1,2, \ldots, s$.

Now $\tilde{S}_{u}$ has the image of the form

$$
\left(\begin{array}{ll}
1 & 0 \\
L_{u} & 1
\end{array}\right)
$$

by conditions (i) and (ii) above; thus we must take $L_{u}=0$. By a simple computation we find then that

$$
\text { (iii) } \quad L_{u}=t_{i}\left(\sum_{k=0}^{p-1} x_{i}^{k}\right)=0
$$

for every relator of category (I) and that

$$
L_{u}=t_{j}\left(x_{i}-1\right)+t_{i}\left(1-x_{j}\right)=0
$$

for every relator of category (II).

At this point we are ready to represent the elements of the subgroup $\mathscr{G}^{\prime} \mid \mathscr{G}^{\prime \prime} \subset \overline{\mathscr{G}}$ by matrices (3.5). For this purpose we introduce the notation

$$
a\left(\sum_{i=1}^{I} \alpha_{i} b_{i}\right)=\prod_{i=1}^{I}\left(b_{i}^{-1} a b_{i}\right)^{\alpha_{i}}
$$

where $a$ and the $b_{i}$ are group elements and the $\alpha_{i}$ are integers. We also note the following:

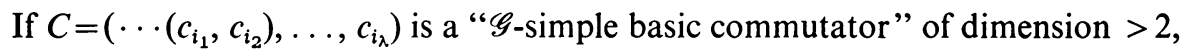
then $C$ is a word in elements $\left(c_{i_{1}}, c_{i_{2}}\right)^{P}$ where $P$ is either $=1$ or a product $c_{j_{1}} c_{j_{2}} \cdots c_{j_{p}}$ with $j_{1} \leqq j_{2} \leqq \cdots \leqq j_{\rho}$ a subsequence of $i_{3} \leqq i_{4} \leqq \cdots \leqq i_{\lambda}$. As a consequence of Lemma 1 and of Definition 2 we obtain then 
LEMMA 2. $\mathscr{G}^{\prime}$ is generated by all elements $\left(c_{i_{1}}, c_{i_{2}}\right)^{P}$ so that

(a) $\left(c_{i_{1}}, c_{i_{2}}\right) \neq 1$ in $\mathscr{G}$,

(b) $P=1$ or $P=c_{j_{1}} c_{j_{2}} \cdots c_{j_{\rho}}$,

(c) $i_{1}>i_{2} \leqq j_{1} \leqq \cdots \leqq j_{\rho}$,

(d) if $i$ occurs $\sigma$ times among $i_{1}, i_{2}, j_{1}, j_{2}, \ldots, j_{\rho}$, then $1 \leqq \sigma<p$,

(e) if $\left(c_{i_{1}}, c_{j_{\mu}}\right)=1$ for $1<\mu \leqq \rho$, then $c_{i_{1}} \geqq c_{j_{\mu}}$.

Since $\left(c_{i_{1}}, c_{i_{2}}\right)^{P}=\left(c_{i_{1}}, c_{i_{2}}\right)\left(\left(c_{i_{1}}, c_{i_{2}}\right), P\right)$ we have at once the corollary:

LEMMA 3. The abelian group $\mathscr{G}^{\prime} \mid \mathscr{G}^{\prime \prime}$ consists of the images of all elements

$$
h=\prod_{i_{1}>i_{2} ;\left(c_{i_{1}}, c_{i_{2}}\right) \neq 1}\left\{\left(c_{i_{1}}, c_{i_{2}}\right)^{\left.Q_{i_{1} i_{2}}\right\}} \text { where } Q_{i_{1} i_{2}}=\sum_{j=1}^{J\left(i_{1}, i_{2}\right)}\left\{\alpha_{i_{1} i_{2} j} P_{i_{1} i_{2} j}\right\},\right.
$$

the $\alpha_{i_{1} i_{2} j}$ are integers and the $P_{i_{1} i_{2} j}$ are elements $P$ as described for $\left(c_{i_{1}}, c_{i_{2}}\right)$ in Lemma 2.

To find the matrices in our representation of $\mathscr{G}^{\prime} / \mathscr{G}^{\prime \prime}$ corresponding to the elements of Lemma 3, let us first compute the ones for the generators of Lemma 2. We obtain easily

$$
\left(c_{i_{1}}, c_{i_{2}}\right)^{P}=\left(\begin{array}{ll}
1 & 0 \\
L & 1
\end{array}\right)
$$

where

$$
\begin{array}{rlrl}
L & =\left[t_{i_{1}}\left(x_{i_{2}}-1\right)+t_{i_{2}}\left(1-x_{i_{1}}\right)\right] \Delta, \\
\Delta & =1 & & \text { for } P=1 \\
& =\prod_{k=1}^{o} x_{j_{k}} & & \text { for } P \neq 1 .
\end{array}
$$

Now a generator $\left(c_{i_{1}}, c_{i_{2}}\right)^{P}$ of Lemma 2 evidently has the following two properties:

(A) If $P \neq 1$, then $\prod_{k=1}^{o} c_{j_{k}}=\prod_{j=1}^{r} c_{j}^{\beta_{1}}$ so that $0 \leqq \beta_{i_{1}}, \beta_{i_{2}} \leqq p-2$.

(B) $\left(c_{i_{1}}, c_{i_{2}}\right) \neq 1$ in $\mathscr{G}$.

Thus $L$ as given by (3.11) has the form

$$
L=\sum_{i=1}^{r} t_{i} Q_{i}\left(x_{1}, x_{2}, \ldots, x_{r}\right)
$$

so that (a) the polynomial $Q_{i}=Q_{i}\left(x_{1}, x_{2}, \ldots, x_{r}\right)$ does not contain a power of $x_{i}$ higher than the $(p-2)$ nd, (b) if $\left(c_{i}, c_{j}\right)=1$ and $Q_{i} \neq 0$ then $1-x_{j}$ does not divide $Q_{i}$. Moreover conditions (A) and (B) imply that the class of polynomials (3.12) which have properties (a) and (b) above and which can be obtained from $L$ by the use of the relations (3.8) and (3.9) consist of $L$ only.

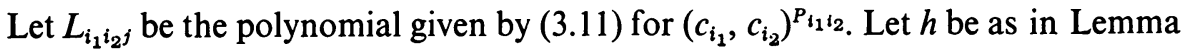
3. Then

$$
\bar{h} \leftrightarrow\left(\begin{array}{cc}
1 & 0 \\
L_{h} & 1
\end{array}\right)
$$


so that $L_{h}$ is the sum

$$
L_{h}=\sum_{i_{1}>i_{2} ;\left(c_{i_{1}}, c_{i_{2}}\right) \neq 1}\left[\sum_{j=1}^{J\left(i_{1}, i_{2}\right)} \alpha_{i_{1} i_{2} j} L_{i_{1} i_{2} j}\right] .
$$

We are now ready to make use of results of Bachmuth [1], here stated as

Lemma 4. Let $\mathscr{F}^{\prime}=[\mathscr{F}, \mathscr{F}], \mathscr{F}^{\prime \prime}=\left[\mathscr{F}^{\prime}, \mathscr{F}^{\prime}\right]$. If $d \in \mathscr{F}^{\prime}$, let $(d)_{\mathscr{F}}$ be its image in $\mathscr{F}$ under the homomorphism $\mathscr{F} \rightarrow \mathscr{F} / \mathscr{F} "=\mathscr{F}$. Then $\mathscr{F}^{\prime} / \mathscr{F}^{\prime \prime}$ is generated by the images, $(f)_{\mathscr{F}}$, of all elements $f=\left(c_{i_{1}}, c_{i_{2}}\right)^{P}$ which constitute the set $\mathscr{S}$. Here $i_{1}>i_{2}$ and $P=\prod_{j=0}^{r} c_{j}^{\gamma_{j}}$ where $c_{0}=1$ and the $\gamma_{j}$ are integers. For $\mathscr{F}$ we have a faithful matrix representation

$$
(d)_{\mathscr{F}} \leftrightarrow\left(\begin{array}{cc}
1 & 0 \\
L_{d} & 1
\end{array}\right)
$$

and in particular

$$
\left(\left(c_{i_{1}}, c_{i_{2}}\right)^{P}\right)_{\mathscr{F}} \leftrightarrow\left(\begin{array}{cc}
1 & 0 \\
L & 1
\end{array}\right)
$$

so that

$$
L=\left[t_{i_{1}}\left(x_{i_{2}}-1\right)+t_{i_{2}}\left(1-x_{i_{1}}\right)\right]\left[\prod_{j=0}^{r} x_{j}^{\gamma_{j}}\right]
$$

where $x_{0}=1$. The only nontrivial relators in $\mathscr{F}^{\prime} / \mathscr{F}^{\prime \prime}$ are products of transforms of Jacobi relators; i.e. they all are consequences of the relations

$$
\left(\left(c_{\alpha}, c_{\beta}\right)^{-1+c_{\gamma}}\right)_{\mathscr{F}}\left(\left(c_{\beta}, c_{\gamma}\right)^{-1+c_{\alpha}}\right)_{\mathscr{F}}\left(\left(c_{\gamma}, c_{\alpha}\right)^{\left.-1+c_{\beta}\right)_{\mathscr{F}}}=1\right.
$$

where $c_{\alpha}, c_{\beta}, c_{\gamma}$ are generators of $\mathscr{F}$ such that $\alpha>\beta$. (We note that a Jacobi relator is a relator corresponding to the Jacobi identity in the associated Lie algebra.)

To apply Lemma 4 we now require more notation. Let $\Phi$ be the multiplicative group of matrices which occur on the right-hand side of $\left(3.13^{\prime}\right)$. Let the homomorphic image $\Psi$ be obtained from $\Phi$ by imposing the relations (3.8) and (3.9), finally let $\Omega$ be the subgroup of $\Psi$ which consists of the matrices on the right-hand side of (3.13).

We note that the polynomial (3.14) is a sum of polynomials (3.12). This means that the coefficient of $t_{i}(i=1,2, \ldots, r)$ in $L_{h}$ does not contain any power of $x_{i}$ higher than the $(p-2)$ nd. We conclude then that a relator of the form (3.8) cannot be a product of elements (3.13) having $L_{h}$ of the form (3.14). We have thus proven that any nontrivial relator in the subgroup $\Omega$ must be a consequence of imposing the relations (3.9) together with the relations (3.15) of Lemma 4 on the group generated by the elements of $\Phi$. Moreover it is evident from the manner in which the relations (3.9) were obtained from the presentation of $\mathscr{G}$ that they are equivalent to imposing the following relations on the group $\mathscr{F}$ :

$$
(f)_{\mathscr{F}}=\left(\left(c_{i_{1}}, c_{i_{2}}\right)^{P}\right)_{\mathscr{F}}=1
$$


if and only if $\left(c_{i_{1}}, c_{i_{2}}\right)=1$ in $\mathscr{G}$. But such elements $\left(c_{i_{1}}, c_{i_{2}}\right)^{P}$ are not among the generators of $\mathscr{G}^{\prime}$ given in Lemma 2. Since our matrix representations are faithful we then easily obtain

LEMMA 5. Any nontricial relator in 'G' $\mathscr{G}^{\prime} \mathscr{G}^{\prime \prime}$ can be found from some nontrivial relator $R$ in $\mathscr{F}^{\prime} / \mathscr{F}^{\prime \prime}$ by imposing the relations (3.16), where $R$ is a word in the generators $(f)_{\mathscr{F}}(f \in \mathscr{S})$.

But the generators $\left(c_{i_{1}}, c_{i_{2}}\right)^{P}$ of $\mathscr{G}^{\prime}$ given in Lemma 2 are evidently images, under the homomorphism $\mathscr{F} \rightarrow \mathscr{G}$, of the elements of a proper subset $\mathscr{T} \subset \mathscr{S}$. To apply Lemma 5 we must now observe the truth of

LEMma 6. Consider the Jacobi relator on the left-hand side of (3.15). Then one of the three conditions holds: (i) All three commutators $\left(c_{\alpha}, c_{\beta}\right),\left(c_{\beta}, c_{\gamma}\right)$ and $\left(c_{\gamma}, c_{\alpha}\right)$ are $=1$ in $\mathscr{G}$. (ii) Not more than one among the commutators $\left(c_{\alpha}, c_{\beta}\right),\left(c_{\beta}, c_{\gamma}\right)$ and $\left(c_{\gamma}, c_{\alpha}\right)=1$ in $\mathscr{G}$, also $c_{\beta} \neq c_{\gamma}$ and $c_{\alpha} \neq c_{\gamma}$. Then there is at least one element $\left(c_{\mu}, c_{\nu}\right)^{c_{\lambda}}$ among $\left(c_{\alpha}, c_{\beta}\right)^{c_{\gamma}}$ and $\left(c_{\gamma}, c_{\alpha}\right)^{c_{\beta}}$ and $\left(c_{\beta}, c_{\gamma}\right)^{c_{\alpha}}$ so that $\left(c_{\mu}, c_{\nu}\right) \neq 1$ in $\mathscr{G}$, but $\left(c_{\mu}, c_{v}\right)^{c_{\lambda}}$ does not occur in the subset $\mathscr{T}$. (iii) $\left(c_{\alpha}, c_{\beta}\right) \neq 1$ in $\mathscr{G}$ and either $c_{\beta}=c_{\gamma}$ or $c_{\alpha}=c_{\gamma}$.

Lemma 6 is easily verified by examining six cases under two sets of mutually exclusive conditions: (A) $\beta<\gamma$ or $\beta>\gamma$. (B) Either all of $c_{\alpha}, c_{\beta}, c_{\gamma}$ do not commute with each other in $\mathscr{G}$, or only the two with the largest subscripts or finally only the two with the smallest subscripts commute. (When the one with the largest subscript commutes with the one with the smallest subscript, then all three commute in the group $\mathscr{G}$ given by (1.3).)

We now note the following:

If $c_{\lambda}=c_{v}$, then

$\left(\left(c_{\lambda}, c_{\mu}\right), c_{v}\right) \equiv\left(c_{\lambda}, c_{\mu}\right)^{-1+c_{\lambda}} \quad \bmod \mathscr{F}^{\prime \prime}, \quad\left(\left(c_{\mu}, c_{v}\right), c_{\lambda}\right) \equiv\left(c_{\lambda}, c_{\mu}\right)^{1-c_{\lambda}} \bmod \mathscr{F}^{\prime \prime}$.

We also recall the manner in which the generators $(a, b)^{P}$ of Lemma 2 were built up from the " $\mathscr{G}$-simple basic commutators" of dimension $>1$. We then find easily that Lemmas 4, 5 and 6 imply

Lemma 7. The images in $\overline{\mathscr{G}}$ of the "G-simple basic commutators" of dimension $>1$ generate $\mathscr{G}^{\prime} \mid \mathscr{G}^{\prime \prime}$ freely.

To proceed from $\mathscr{G}^{\prime} \mid \mathscr{G}^{\prime \prime}$ to $\mathscr{G}^{\prime}$ we next prove

LEMMA 8. $\mathscr{G}^{\prime}$ is a free group.

Proof. $\mathscr{G}$ is the free product of abelian groups $\mathscr{G}(1), \mathscr{G}(2), \ldots, \mathscr{G}(s)$. By the "Kurosh subgroup theorem" ([3], [6])

$$
\mathscr{G}^{\prime}=V * \prod_{j}^{*} x_{j}^{-1} U_{j} x_{j}
$$

where $V$ is a free group and each $x_{j}^{-1} U_{j} x_{j}$ is the conjugate of a subgroup $U_{j}$ of one of the free factors $\mathscr{G}(i)$. But such a factor or any conjugate of it is abelian and therefore intersects $\mathscr{G}^{\prime}$ in the identity only. Hence $\mathscr{G}^{\prime}=V$. 
Thus $\mathscr{G}^{\prime}$ has the set $\mathscr{A}=\left\{w_{i}\right\}$ of free generators. Let $\alpha$ be the cardinality of $\mathscr{A}$. Let $v_{1}, v_{2}, \ldots, v_{N}$ be the " $\mathscr{G}$-simple basic commutator" of dimension $>1$ in some order. First suppose $\alpha<N$. Then the images $\bar{w}_{1}, \bar{w}_{2}, \ldots, \bar{w}_{\alpha}$ of the set $\mathscr{A}$ in $\overline{\mathscr{G}}$ are free generators of the abelian group $\mathscr{G}^{\prime} / \mathscr{G}^{\prime \prime}$ in contradiction to Lemma 7 and the well-known fact ([3], [6]) that any two bases of a finitely generated free abelian group contain the same number of elements. Hence $\alpha \geqq N$. If $\alpha>N$, consider the set $\mathscr{B}$ consisting of $v_{1}, v_{2}, \ldots, v_{N}$ together with $\alpha-N$ words in the $v_{j}$. Then $\mathscr{B}$ has cardinality $\alpha$ and is a set of generators of $\mathscr{G}^{\prime}$; here $\alpha$ is finite by Lemma 1 . But it is well-known ([3], [6]) that if $\mathscr{A}$ generates the group $V$ freely and $\mathscr{B}$ is a set of generators of cardinality $\alpha$, then $\mathscr{B}$ is also a set of free generators. Hence $\alpha=N$ and the $v_{1}, v_{2}, \ldots, v_{N}$ are free generators. Theorem 2 has been established.

\section{REFERENCES}

1. S. Bachmuth, Automorphisms of free metabelian groups, Trans. Amer. Math. Soc. 118 (1965), 93-104. MR 31 \#4831.

2. K. W. Gruenberg, Residual properties of infinite soluble groups, Proc. London Math. Soc. (3) 7 (1957), 29-62. MR 19, 386.

3. Marshall Hall, Jr., The theory of groups, Macmillan, New York, 1959. MR 21 \#1996.

4. Phillip Hall, A contribution to the theory of groups of prime power order, Proc. London Math. Soc. (2) 36 (1934), 29-95.

5. Wilhelm Magnus, On a theorem of Marshall Hall, Ann. of Math. 40 (1939), 764-768. MR 1, 44.

6. Wilhelm Magnus, Abraham Karrass and Donald Solitar, Combinatorial group theory: Presentations of groups in terms of generators and relations, Pure and Appl. Math., vol. 13, Interscience, New York, 1966. MR 34 \#7617.

7. Ruth Rebekka Struik, On nilpotent products of cyclic groups. II, Canad. J. Math. 13 (1961), 557-568. MR 26 \#2486.

8. Hermann V. Waldinger, The lower central series of groups of a special class, J. Algebra 14 (1970), 229-244. MR 41 \#5502.

Department of Mathematics, Polytechnic Institute of Brooklyn, Brooklyn, New YORK 11201 